\title{
Beauty production in DIS and the measurement of $F_{2}{ }^{b b}$ at ZEUS
}
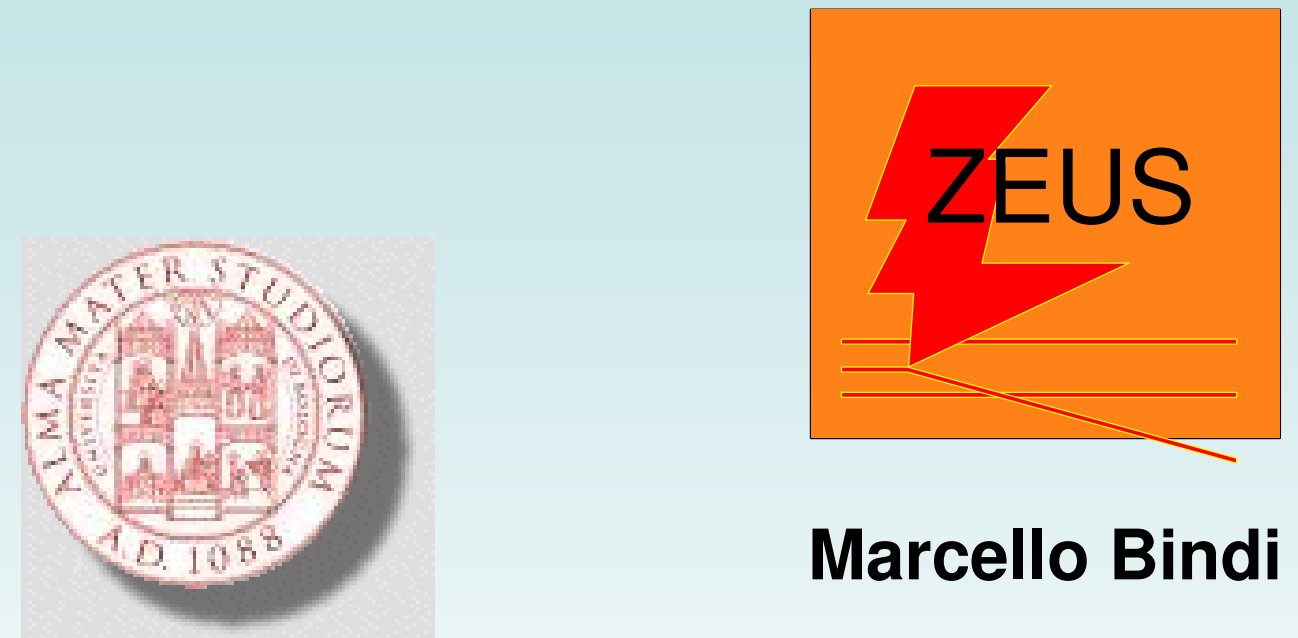

Marcello Bindi

University and INFN of Bologna on behalf of the ZEUS Collaboration

DIS 2009, 26-30 April 2009, Universidad Autonoma de Madrid XVII International Workshop on Deep-Inelastic Scattering and Related Subjects 


\section{Motivations}

- Heavy flavour production at HERA can be studied for different kinematic regions, from Photoproduction to DIS and for different values of transverse momentum of the heavy quark.

- In DIS regime the heavy quarks are produced mainly by the Boson-Gluon-Fusion process (LO).

- This process is directly sensitive to gluon content inside the proton: possible constraint on $\mathrm{g}(\mathrm{x})$ in PDF fits.

- PDFs: $F_{2}{ }^{\text {bb }}$ measurements at high $Q^{2}$ important for LHC e.g. $b b->H$

- Important test of $\mathrm{pQCD}$ at different scales $\left(M_{Q}, p^{\top}{ }_{Q}, Q^{2}\right)$.

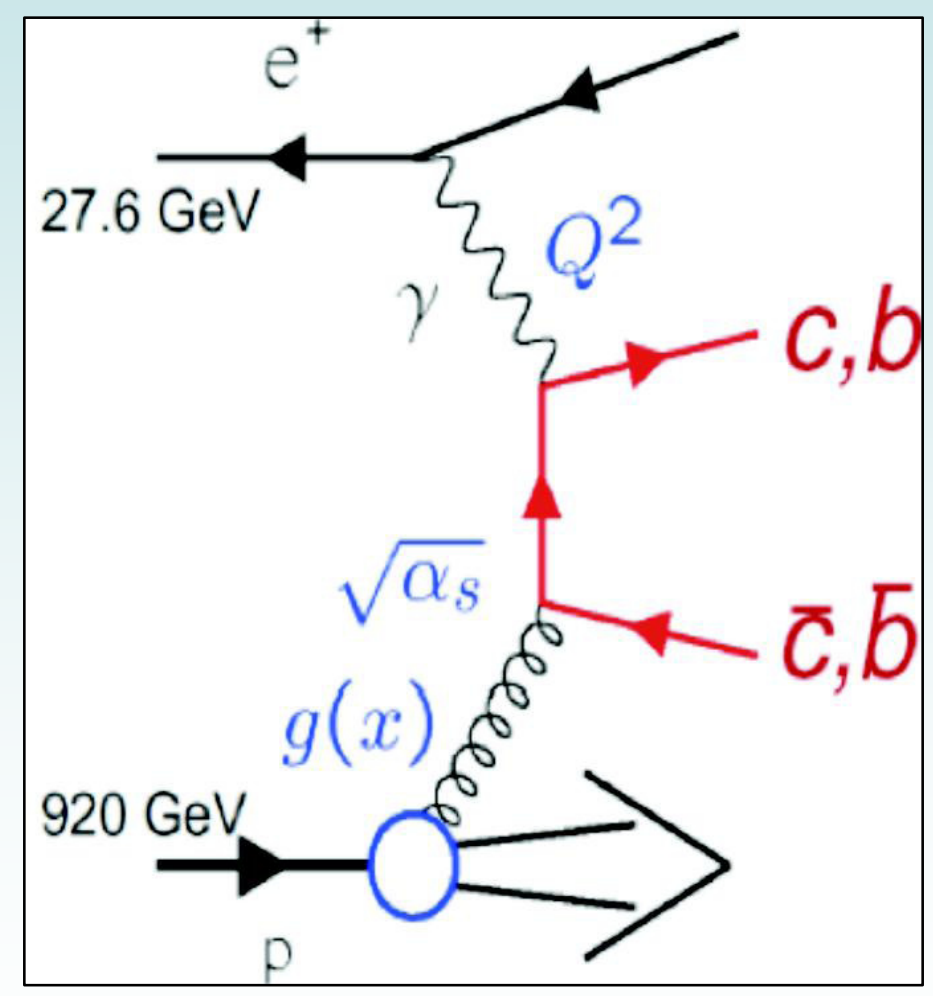




\section{Test of theoretical models at NLO}

Massive approach (Fixed Flavour Number Scheme): FFNS

- c \& b massive $\rightarrow$ full massive matrix elements; DIS : Harris \& Smith, HVQDIS

- appropriate for $\mathrm{Q}^{2} \sim \mathrm{M}_{\mathrm{Q}}^{2}$ fully differential NLO program

c \& b produced dynamically in the hard subprocess

(not part of the proton; 3 active flavours in proton: $u, d, s$ )

Massless approach (Zero Mass Variable Flavour Number Scheme): ZM-VFNS

- $\quad$ \& b massless $\rightarrow$ resums $\left[\alpha_{S} \ln \left(Q^{2} / M^{2}{ }_{Q}\right)\right]^{n}$;

- appropriate for $Q^{2}>>M_{Q}{ }^{2}$

DIS : only inclusive calculation

$\Rightarrow$ c \& b present in proton of $F_{2}{ }^{Q Q}$ available

Combined approach (Generalized Mass Variable Flavour Number Scheme):

- equivalent to massive at small $\mathrm{Q}^{2}$

GM-VFNS

- equivalent to massless at high $\mathrm{Q}^{2}$ DIS : only $F_{2}{ }^{Q Q}$ available 


\section{Beauty at ZEUS.}

Beauty fraction in DIS very low (< 1\%); by selecting events with muons we can reach a fraction of $\sim 10 \%$. How to distinguish beauty component from charm and light flavour?

- $\mathbf{p}_{\mathrm{T}}{ }^{\text {rel }}: \mathrm{p}_{\mathrm{T}}$ of the muon relative to the associated jet axis

- $\delta$ : impact parameter of the muon w.r.t. the "beam spot" in $\mathrm{X}, \mathrm{Y}$ plane. Sign from muon-jet association.

$\rightarrow$ vertex detector with good resolution needed (MVD).
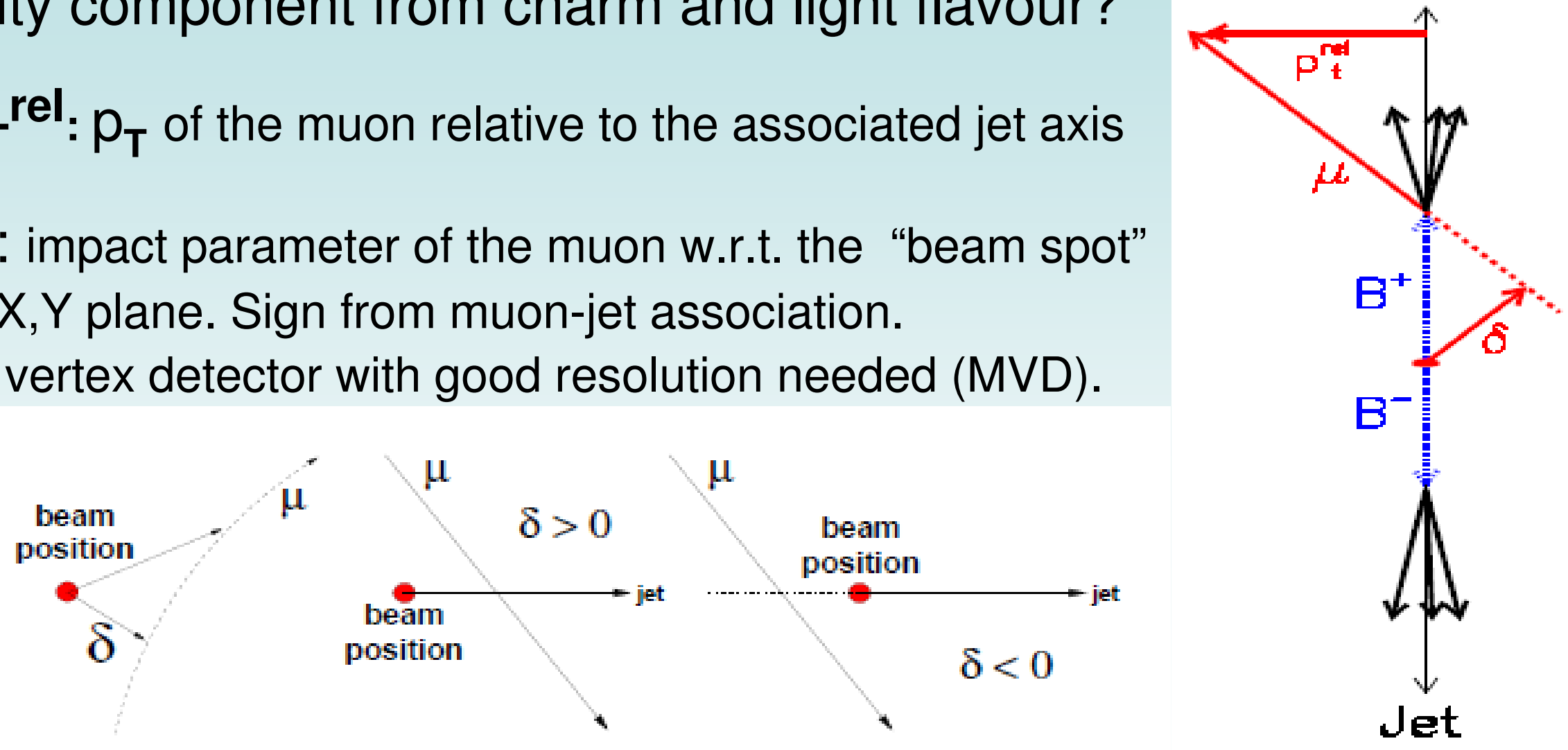

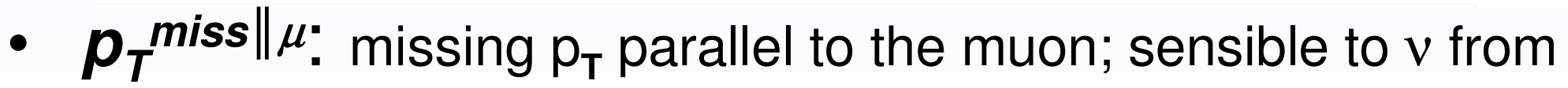
semi-leptonic decay $\rightarrow$ high resolution hadronic $C a l$ needed. 


\section{ZEUS measurements}

HERA- I ZEUS measurement of $b$ in DIS used $p_{T}{ }^{\text {rel }}$ to distinguish $\mathrm{b}$ from $\mathrm{c} / \mathrm{lf}$ and required an hard jet to increase the $b$ fraction; charm content was taken from other measurements.

DESY-04-070, Physics Letters B 599 (2004)

- c and b are extracted simultaneously;

- use also muon impact parameter with respect to primary vertex (beamspot) from MicroVertexDetector and $\mathbf{p}_{\mathrm{T}}$ balance from neutrinos.

http://arxiv.org/abs/0904.3487

DESY-09-056 zeus-pub-09-003 


\section{Beauty \& Charm from muons}

- New measurement uses first part of HERA II data 2005e $\rightarrow L=126$ pb-1

Selection cuts:

- $\mathrm{Q}^{2}>20 \mathrm{GeV}^{2}$

$\cdot 0.01<\mathrm{y}<0.7$

$--1.6<\eta^{\mu}<2.3$

- $p_{\mathrm{T}}^{\mu}>1.5 \mathrm{GeV}$

- Anti-isolation cut:

$\mathrm{E}($ cone, $\mathrm{R}=1)>0.5 \mathrm{GeV}$

- jet-mu association required via $k_{\mathrm{T}}$ algorithm, $\mathrm{p}_{\mathrm{T}}{ }^{\mathrm{jet}}>2.5 \mathrm{Gev}$

Final sample : 11126 MUONS

\section{ZEUS}
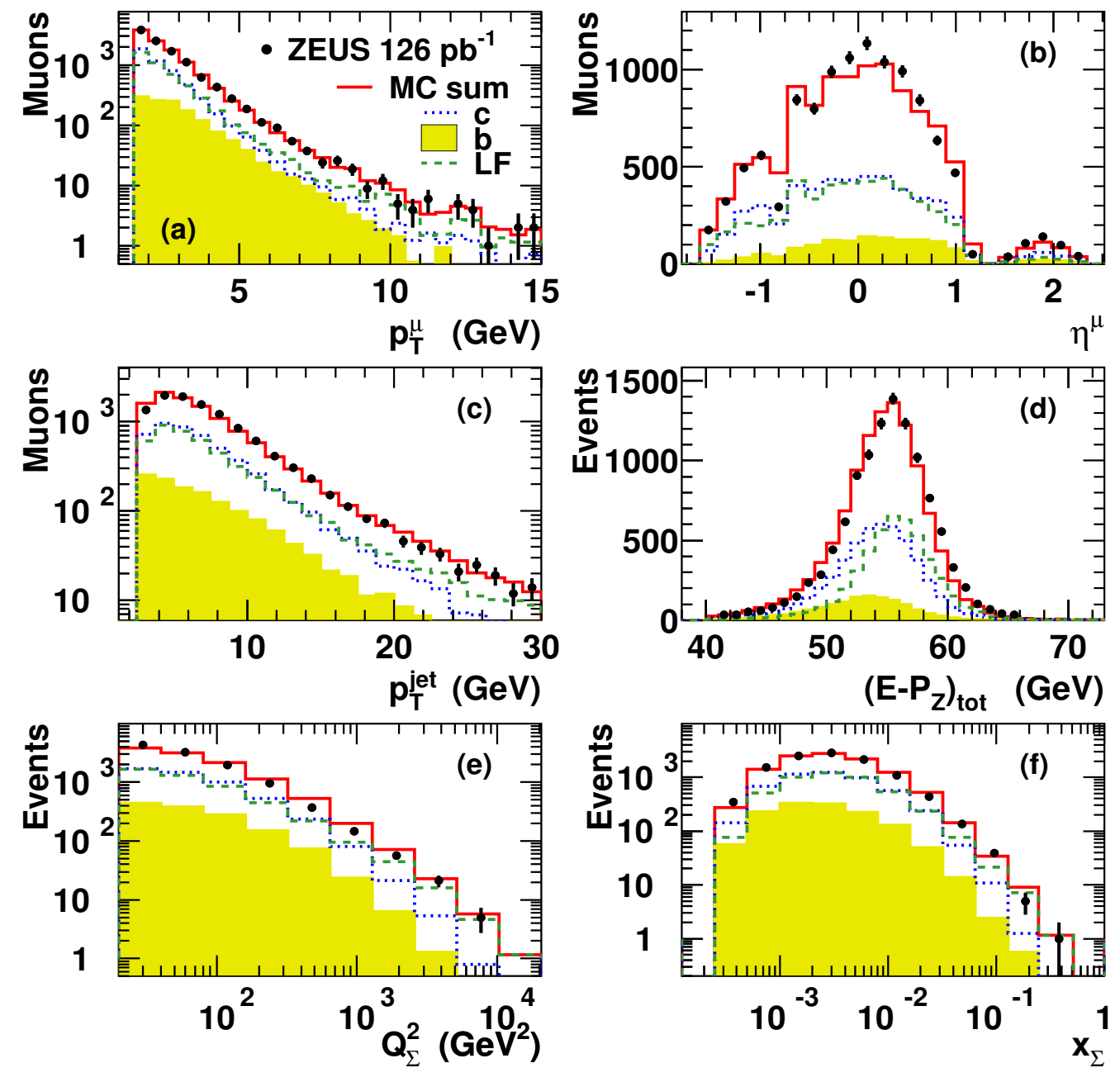

6 
ZEUS
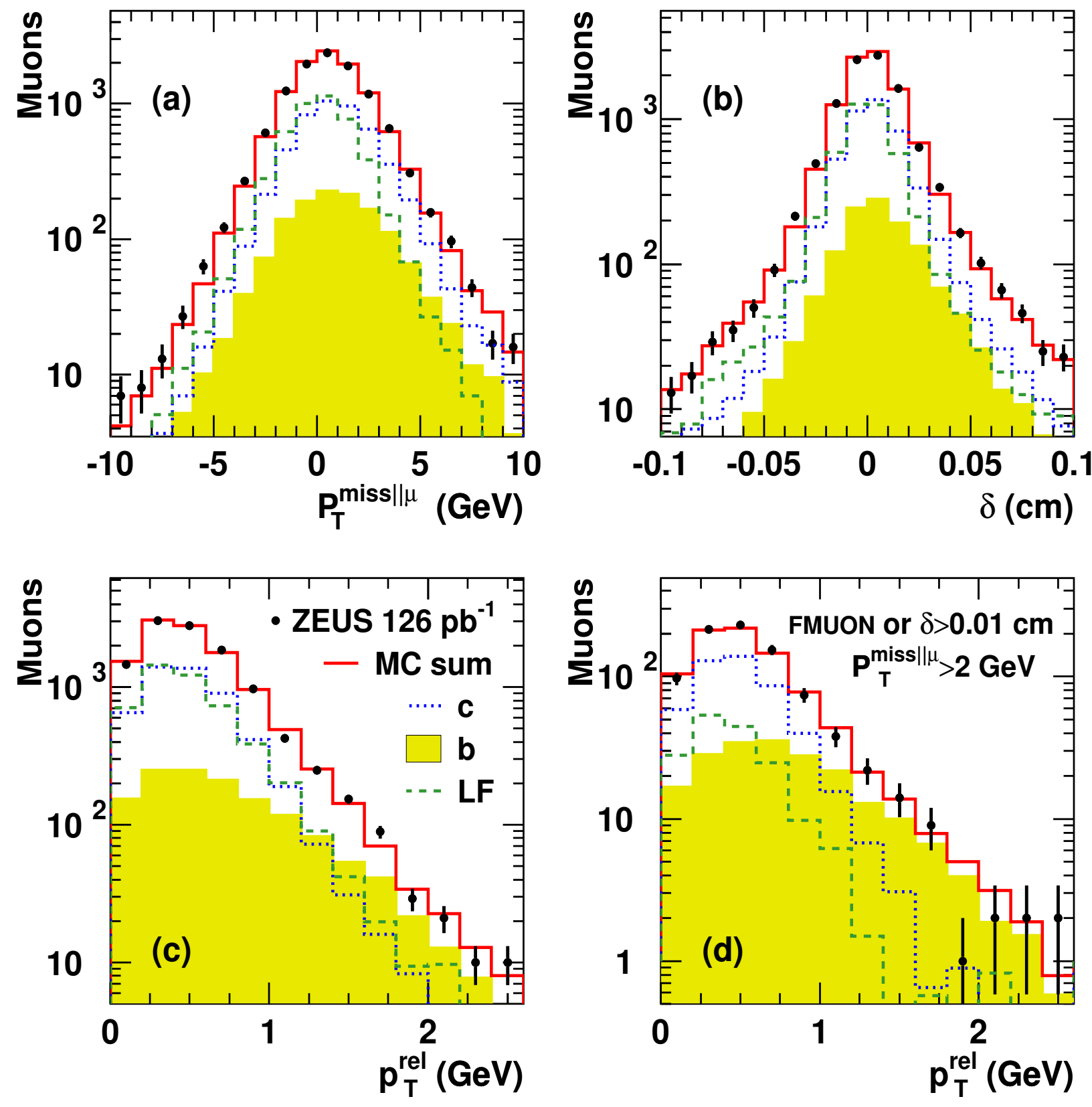

Fits control plots

- 3D simultaneous fit of discriminating variables sensitive to different aspects of $\mathrm{HQ}$ decays.

- MC templates from RAPGAP (charm and beauty) and MEPS (light flavours).

- Background templates for discriminating variables corrected to describe data; inclusive DIS sample has been used. 


\section{ZEUS: NLO QCD predictions for beauty}

- The HVQDIS program has been used to evaluate cross sections for heavy quark production at NLO $\left(O\left(\alpha_{s}^{2}\right)\right)$ in the Fixed Flavour Number Scheme (the only available).

\begin{tabular}{|c|c|c|}
\hline Quantity & Value & Variation \\
\hline $\begin{array}{c}\text { Renormalisation \& } \\
\text { Factorisation scale }\left(\mu_{R}, \mu_{\mathrm{F}}\right)\end{array}$ & $\mu_{\boldsymbol{R}}=\mu_{\mathrm{F}}=\sqrt{Q^{2}+4 M_{c}^{2}}$ & $\begin{array}{c}2 \sqrt{Q^{2}+4 M_{x}^{2}} \\
\frac{1}{2} \sqrt{Q^{2}+4 M_{x}^{2}}\end{array}$ \\
\hline Peterson Parameter $\left(\varepsilon_{\mathbf{b}}\right)$ & $\mathbf{0 . 0 0 3 5}$ & \pm 0.002 \\
\hline Beauty Mass $\left(\mathbf{M}_{\mathbf{b}}\right)$ & $\mathbf{4 . 7 5} \mathrm{GeV}$ & $\pm 0.25 \mathrm{GeV}$ \\
\hline \hline Input PDF & Zeus NLO PDF & $\begin{array}{c}\text { Upper and lower predictions } \\
\text { of ZEUS NLO PDF }\end{array}$ \\
\hline Branching Ratio & $\mathbf{0 . 2 0 9}$ & $\pm \mathbf{0 . 0 0 4}$ \\
\hline
\end{tabular}

- Biggest uncertainty from $\mathbf{M}_{\mathbf{B}}$ and from $\boldsymbol{\mu}_{\mathbf{R}}$. Uncertainties added in quadr. 26-30 April 2009 DIS 2009, UAM 


\section{Total cross sections}

\section{$Q^{2}>20 \mathrm{GeV}^{2} ; 0.01<y<0.7$;}

$p_{T}^{\mu}>1.5 \mathrm{GeV},-1.6<\eta^{\mu}<2.3$

\section{- NLO cross sections:}

$\sigma_{\text {c,th }}=184_{-40}^{+26} \mathrm{pb}$

$\sigma_{b, t h}=33_{-5}^{+5} \quad \mathrm{pb}$

- Main syst. Uncertainties:

charm: $\boldsymbol{p}_{T}^{\text {miss }}$ calibration, $M C$ model; $\quad$ beauty: $\delta, p_{T}{ }^{\text {rel }}, M C$ model

\section{- Global fractions:}

FC $=0.456 \pm 0.029$ (stat.)

$\mathrm{Fb}=0.122 \pm 0.013$ (stat.)

\section{- Total cross sections:}

$$
\begin{aligned}
& \sigma_{\mathrm{c}}=164 \pm 10 \text { (stat.) }{ }_{-31}^{+30} \text { (syst.) pb } \\
& \sigma_{\mathrm{b}}=63 \pm 7 \text { (stat.) }{ }_{-11}^{+18} \text { (syst.) pb } \\
& \rho_{c b}=-0.43
\end{aligned}
$$

3D fit calculated for each bin of $\mathbf{Q}^{2}, \mathbf{x}, \mathbf{p}_{\mathbf{T}}{ }^{\mu}$, eta ${ }^{\mu} \rightarrow$ differential cross sections. 


\section{ZEUS}
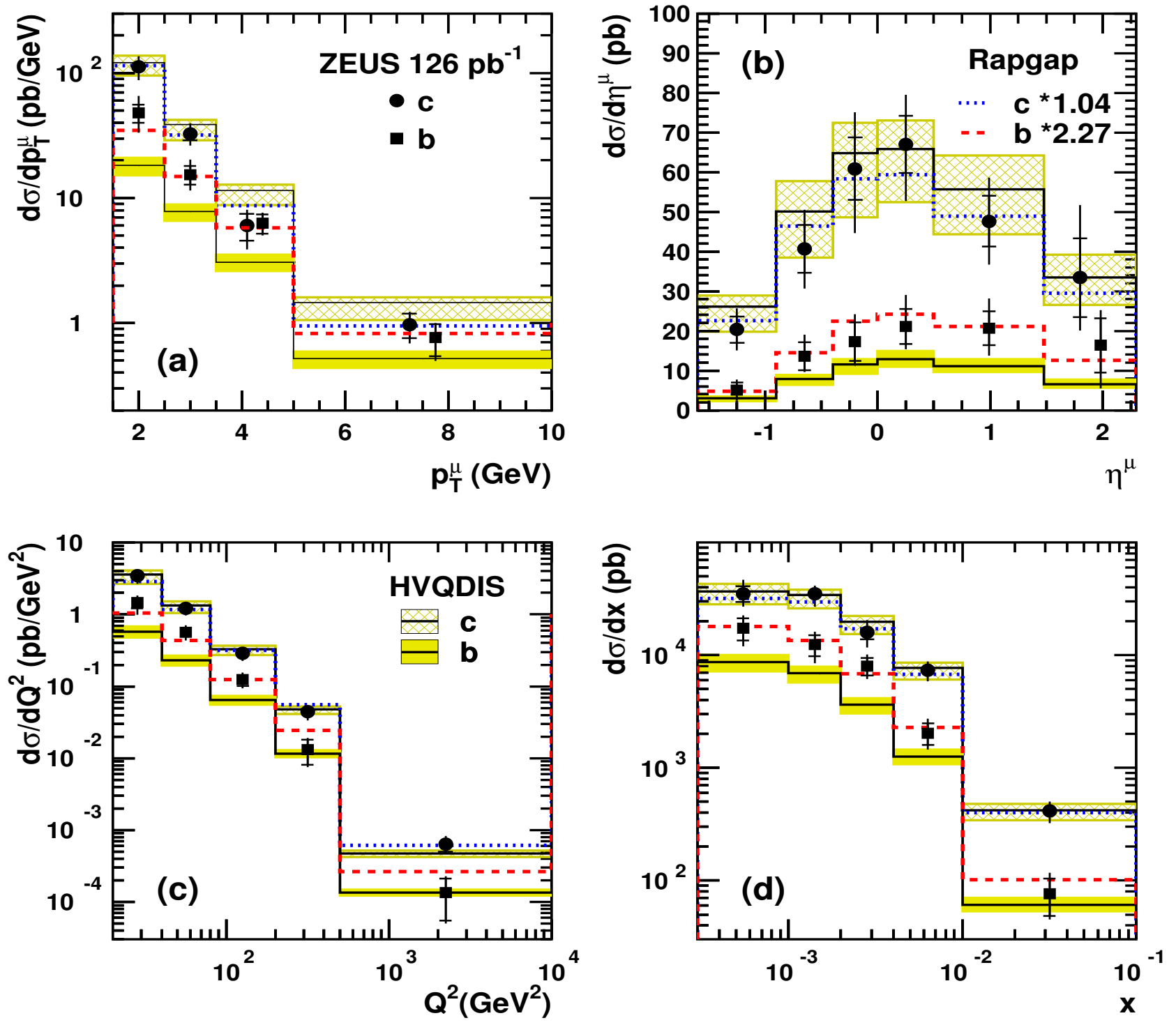

Differential cross sections

- charm: good agreement with HVQDIS and RAPGAP.

- beauty: excess at low $Q^{2}$ (within $\sim 2 \sigma$ the significance). 


\section{Extraction of $F_{2}{ }^{b b}$}

$$
\frac{d^{2} \sigma^{q \bar{q}}}{d x d Q^{2}}=\mathcal{K}\left[F_{2}^{q \bar{q}}\left(x, Q^{2}\right)-\frac{y^{2}}{Y_{+}} F_{L}^{q \bar{q}}\left(x, Q^{2}\right)\right]=\mathcal{K} \tilde{\sigma}^{q \bar{q}}\left(x, Q^{2}, s\right)
$$$$
\mathcal{K}=Y_{+}\left(2 \pi \alpha_{\text {em }}^{2}\right) /\left(x Q^{4}\right) \quad Y_{+}=1+(1-y)^{2}
$$

Beauty NLO

$$
F_{2}^{q \bar{q}}\left(x, Q^{2}\right)=\sigma^{q} \frac{F_{2}^{q \bar{q}, \text { th }}\left(x, Q^{2}\right)}{\sigma^{q, \text { th }}},
$$

\section{Calculated at NLO in} FFNS using HVQDIS

- Extrapolation factor to the full muon phase space.

- Branching ratio $q \rightarrow \mu$.

- Bin centering.

- Correction for the $F_{L}{ }^{q q}$ (1- 4\%).

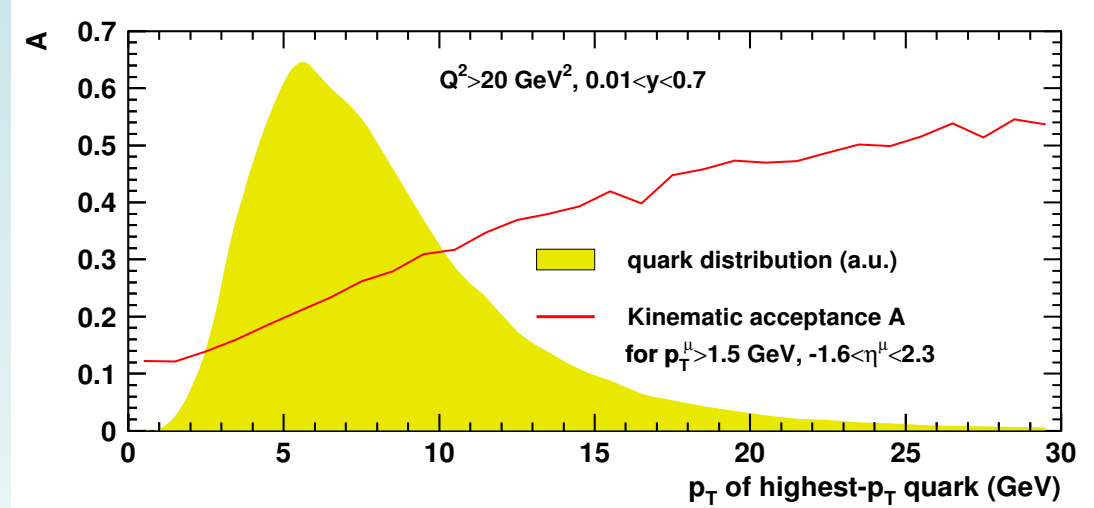

- QED radiation correction. 26-30 April 2009

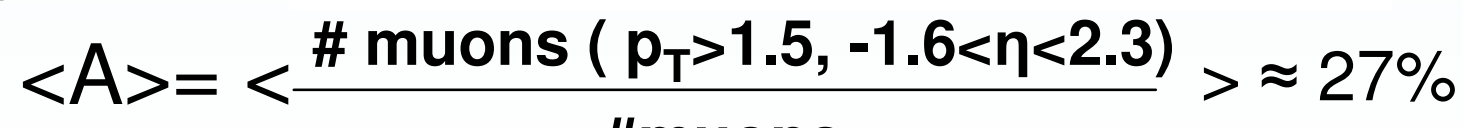

$$
\begin{aligned}
& \text { DIS 2009, UAM \#muons }
\end{aligned}
$$




\section{ZEUS}
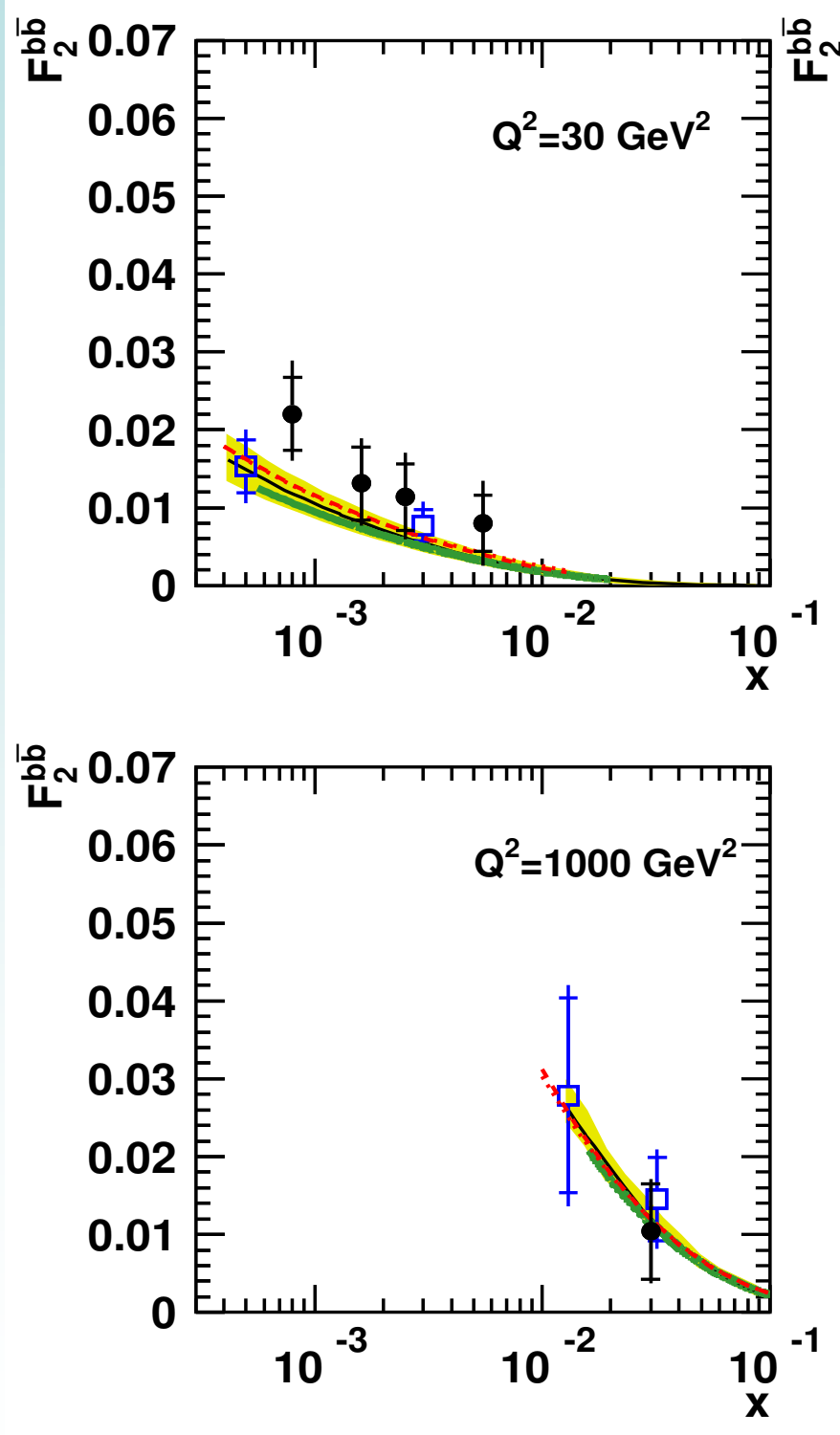

26-30 April 2009

\section{$F_{2}^{b b}(\mathrm{x}):$}

published

data

\section{VS}

- ZEUS 126 pb $^{-1}(\mu)$

ㅁ H1 $57 \mathrm{pb}^{-1}$ (VTX)

different theories 


\section{ZEUS}

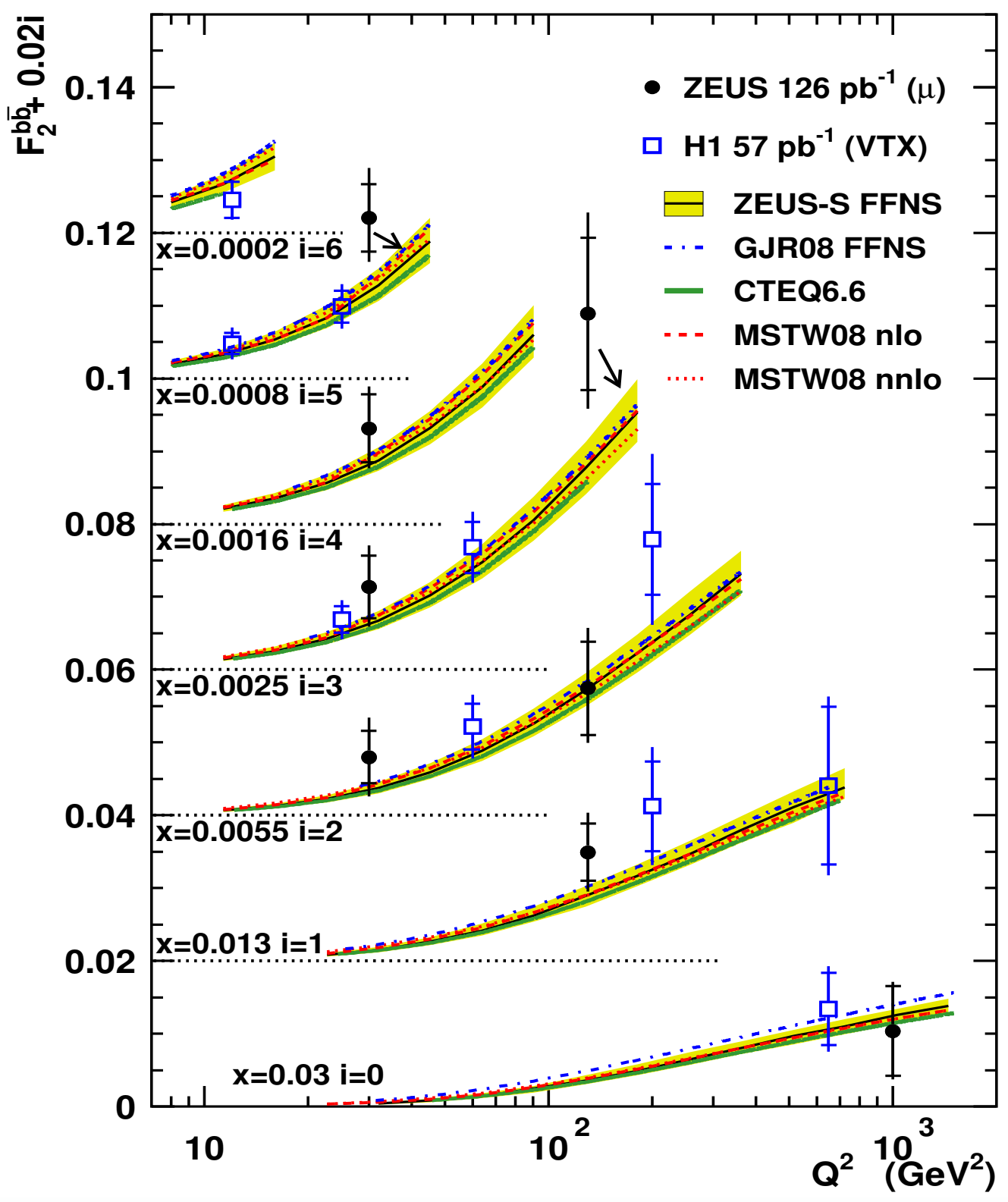

\section{$\mathrm{F}_{2}{ }^{b b}$ at HERA}

- $\mathrm{F}_{2}{ }^{\mathrm{bb}}$ determined at ZEUS with part of HERA II data (1/3 lumi) for the first time.

- The published measurements cannot distinguish between different gluon parameterizations.

- ZEUS and H1 measurements are in good agreement.

-Theoretical uncertainty smaller for beauty than for charm. 


\section{HERA}

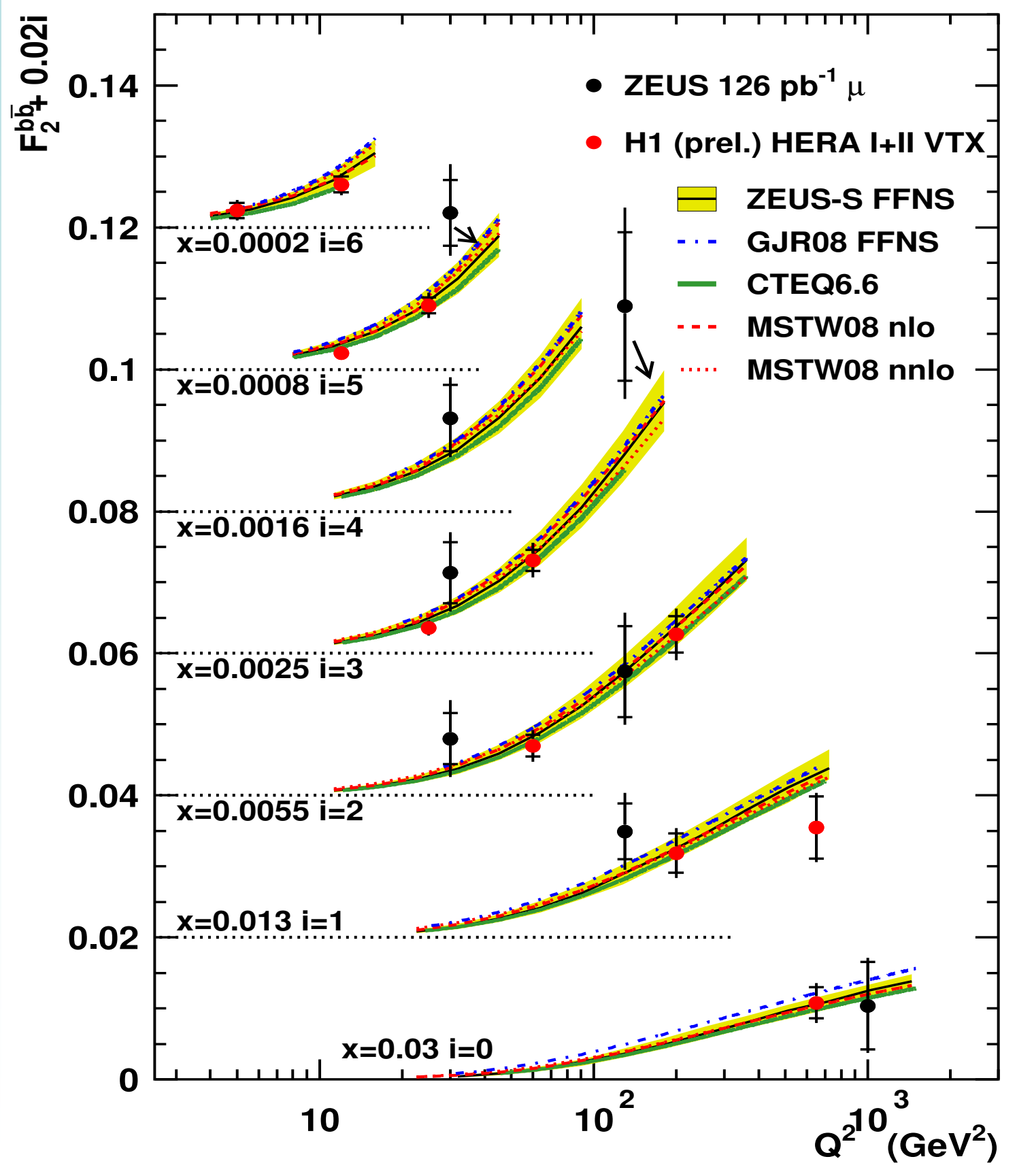

- $F_{2}{ }^{b b}$ determined at ZEUS with part of HERA II data (1/3 lumi) for the first time.

- Good agreement between theory and data.

-H1 preliminary points could distinguish between different theories? 


\section{Conclusions and outlook}

- The beauty contribution to the proton structure functions, $F_{2}$, has been measured at ZEUS for the first time with HERAll data using new techniques.

- The two collaborations, ZEUS and H1, using very different methods for the analysis, implying different extrapolations factors, agree on the results.

- The precision of the new measurement is good, specially for higher $Q^{2}$ region.

- The use of the whole HERA data sample could really help in constraining the gluon parameterization in the proton. 


\section{Backup}




\section{HERA}

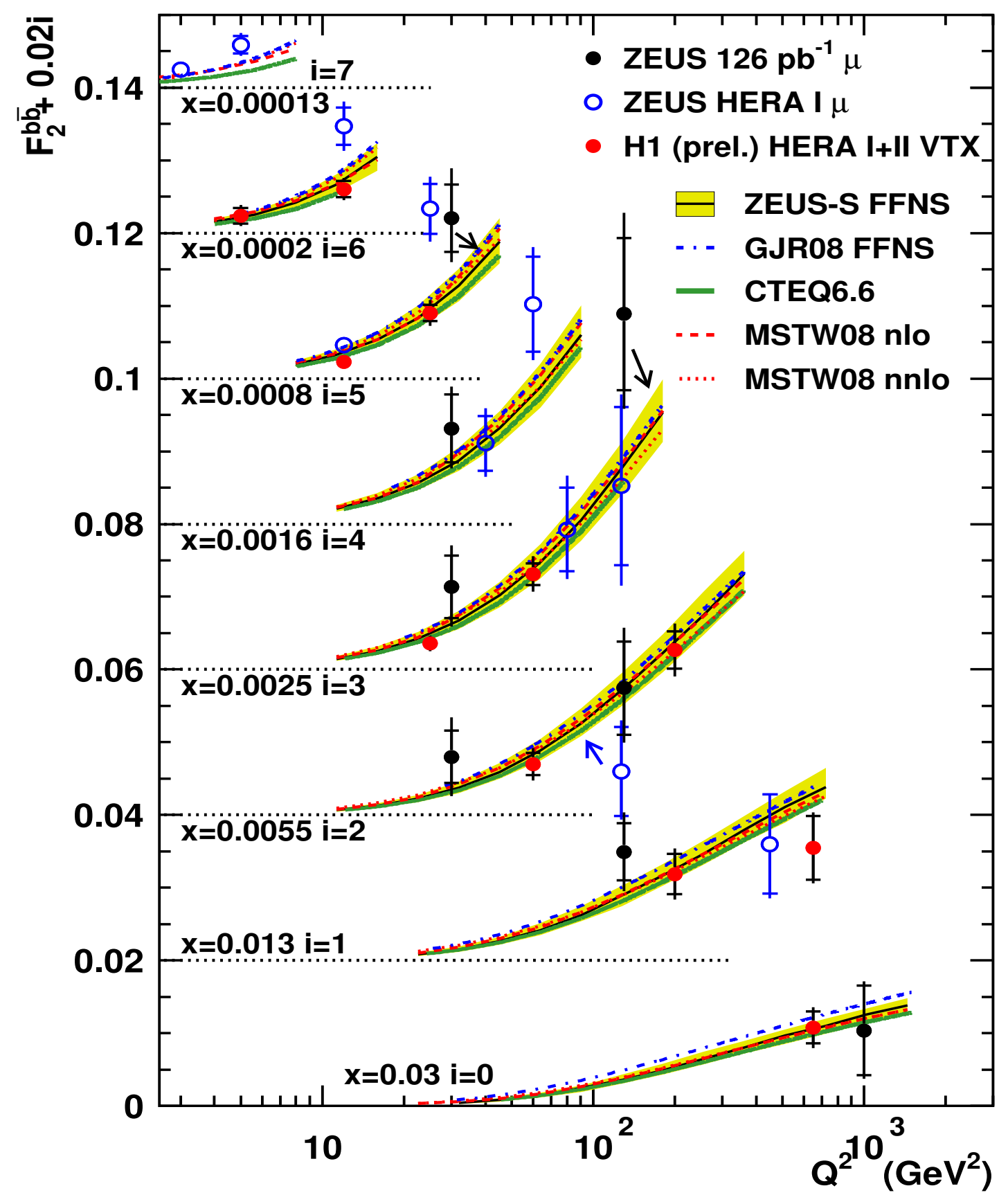

\section{$F_{2} b b$ (publ.+prel.)}

- $F_{2}{ }^{b b}$ determined at ZEUS with part of HERA II data (1/3 lumi).

- Good agreement between theory and data.

- Good precision for high $\mathrm{Q}^{2}$ point.

-H1 preliminary points could distinguish between different theories? 
ZEUS
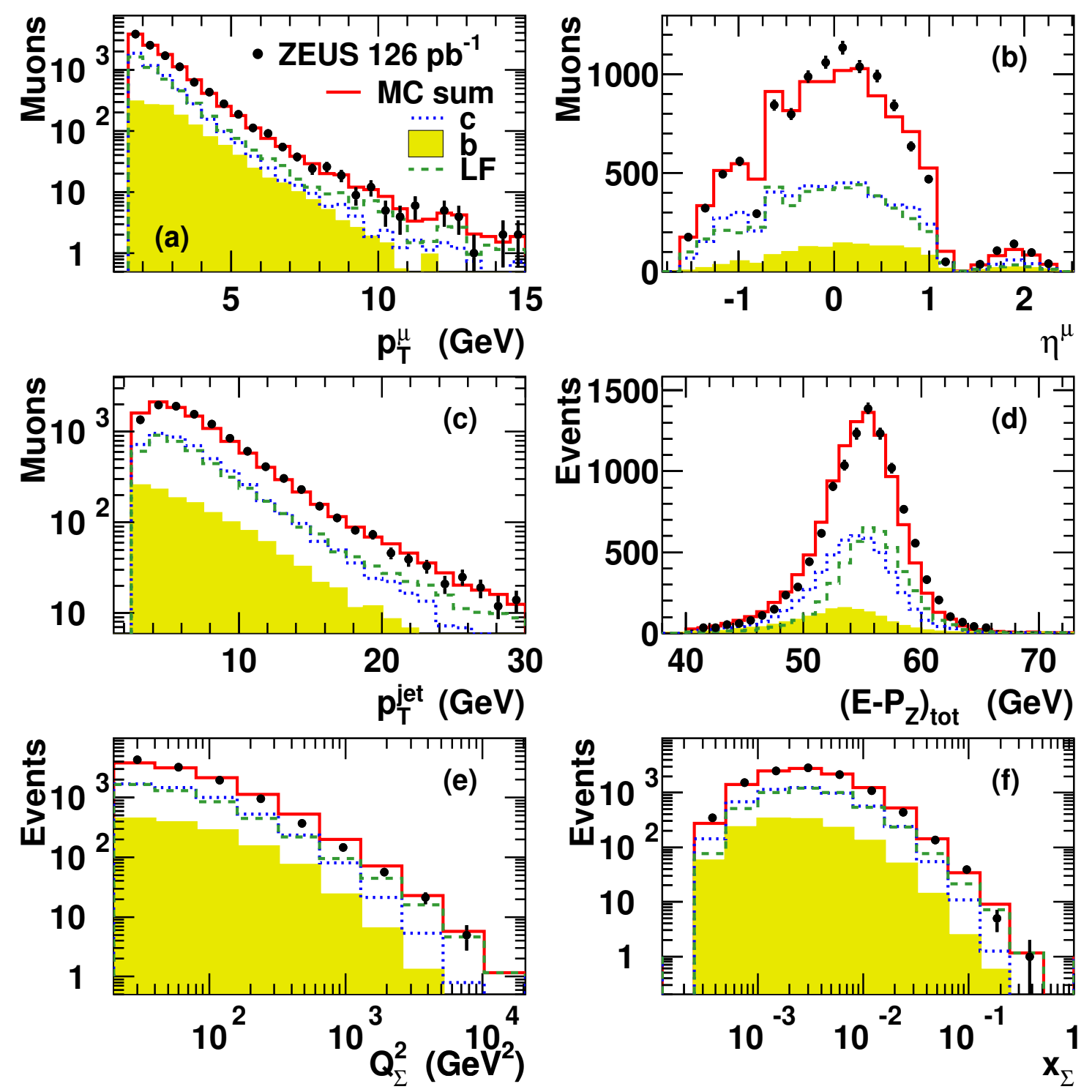

26-30 April 2009

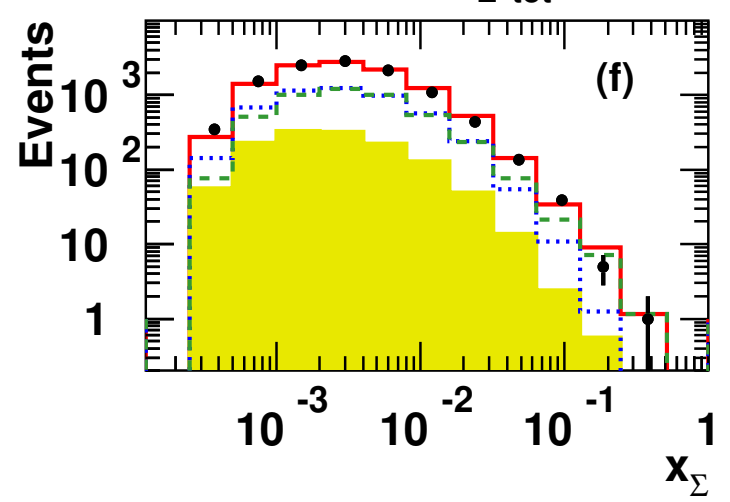

DIS 2009, UAM

\section{Main variables control plots}

$\boldsymbol{A}_{\mathrm{c}(\mathrm{b})}$ varies from $\approx 23 \%$ (16\%) to $\approx 35 \%(25 \%)$ for $\mathrm{p}_{\mathrm{T}}{ }^{\mu}<$ or $>2.5 \mathrm{GeV}$ 


\section{All systematic uncertainties/1}

1. B/RMUON efficiency: it was varied by its uncertainty of on average $\pm 5 \%(\mp 5, \mp 5) \%$;

2. FMUON efficiency: it was varied by $\pm 20 \%(\mp 2, \mp 5) \%$;

3. "false muon" probability: it was varied within the corresponding uncertainty $\left(\begin{array}{c}-3 \\ +4\end{array}, \mp 1\right) \%$;

4. global energy scale: it was varied by $\pm 2 \%\left(\begin{array}{c}-4-3 \\ +5 \%+2\end{array}\right) \%$;

5. calibration of $p_{T}^{\text {miss }} \mid \mu$, it was evaluated by varying the hadronic transverse momentum in the $\mathrm{MC}$ by $\pm 0.1 \mathrm{GeV}$, as allowed by the transverse momentum balance in the control sampl $( \pm 12,-2) \%$

6. hadronic energy resolution: it was varied in the $\mathrm{MC}$ by $\pm 5 \%$ as allowed by the transverse momentum balance in the control sample $\left(\begin{array}{c}+1 \\ +2\end{array} 77\right) \%$;

7. simulation of the tails of miss $p_{T}^{\text {m }}$ : the fits were redone in the restricted range $\left|p_{T}^{\text {miss }}\right||\mu|<$ $5 \mathrm{GeV}(0,-6) \% ;$

8. resolution on $\delta$ : the smearing applied to the $\mathrm{MC}$ was varied by $\pm 25 \%$ as allowed by the control sampl $\left(\begin{array}{c}-3+11 \\ +2 i-9\end{array}\right) 9$

9. $p_{T}^{\mathrm{rel}}$ shape of LF and charm: it was evaluated by varying the $p_{T}^{\mathrm{rel}}$ correction by $\pm 50 \%$ $(-1.5,+8,5)$ 


\section{All systematic uncertainties/2}

10. hadronic energy flow near the muon: it was evaluated by varying the cut on $E^{\text {iso }}$ by ${ }_{-0.25}^{+0.50} \mathrm{GeV}(0,-1) \%$;

11. jet fragmentation: the cut on $p_{T}^{\text {jet }}$ was varied by $\pm 0.5 \mathrm{GeV}( \pm 2.5,+2.5) \%$;

12. charm SL decay spectrum: the reweighting to the CLEO model was varied by $\pm 50 \%$, $\left(\begin{array}{c}-4+3 \\ +3 ;-2\end{array}\right) \%$

Charm

Beauty

13. MC model dependence: RAPGAP was reweighted to reproduce the measured differential cross sections in $Q^{2}$ or in $p_{T}^{H}$ and the largest deviation from the nominal cross section was taken $+6,+207 \%$

14. higher order effects: this uncertainty was evaluated by varying the HQ distribution before parton showering in RAPGAP by the difference between NLO and leading order, as evaluated with HvQD $\left(\begin{array}{l}+6+2 \\ -10 ;-3\end{array}\right) \%$

15. MVD efficiency: the efficiency of the cut on the number of MVD hits was varied by its uncertainty $(\mp 3, \mp 3) \%$;

16. CTD siumlation: tracks were required to pass $\geq 4$ superlayers in the B/RMUON region and to have $\geq 7$ hits in the FMUON region $(+1,0) \%$;

Total systematic

uncertainty

C B

$\left(\begin{array}{l}+18 \\ -19\end{array},+28\right) \%$

17. integrated luminosity: measurement uncertainty $(\mp 2.6, \mp 2.6) \%$. 


\section{Theoretical models used}

\section{GM-VFNS}

\section{FFNS}

\section{ZEUS-S}

- calclated with HVQDIS

- NLO $O\left(\alpha_{s}^{2}\right)$

- $m_{c}=1.5 \pm 0.2 \mathrm{GeV}$, $m_{b}=4.75 \pm 0.25 \mathrm{GeV}$

- $\mu_{0}=\sqrt{4 m^{2}+Q^{2}}$, $\mu_{0} / 2<\mu_{F}<2 \mu_{0}$, $\mu_{0} / 2<\mu_{R}<2 \mu_{0}$

- ZEUS-S-FF PDF (with expt. uncert.)

\section{GJR08}

(Eur.Phys.J.C (2008) 355)

- grids from authors

- NLO $O\left(\alpha_{s}^{2}\right)$

- $m_{c}=1.3 \mathrm{GeV}$, $m_{b}=4.2 \mathrm{GeV}$

- $\mu_{R}=\mu_{F}=m_{q}$

\section{MSTW08 nlo, nnlo}

(arXiv:0901.0002)

- prel. code from authors

- NLO: $O\left(\alpha_{s}^{2}\right)$ @low $Q^{2}$, $O\left(\alpha_{s}\right)$ ehigh $Q^{2}$

- NNLO: approx. $O\left(\alpha_{s}^{3}\right) @ \operatorname{low} Q^{2}$, $O\left(\alpha_{s}^{2}\right)$ ehigh $Q^{2}$

- $m_{c}=1.4 \mathrm{GeV}$, $m_{b}=4.75 \mathrm{GeV}$

- $\mu_{R}=\mu_{F}=Q$

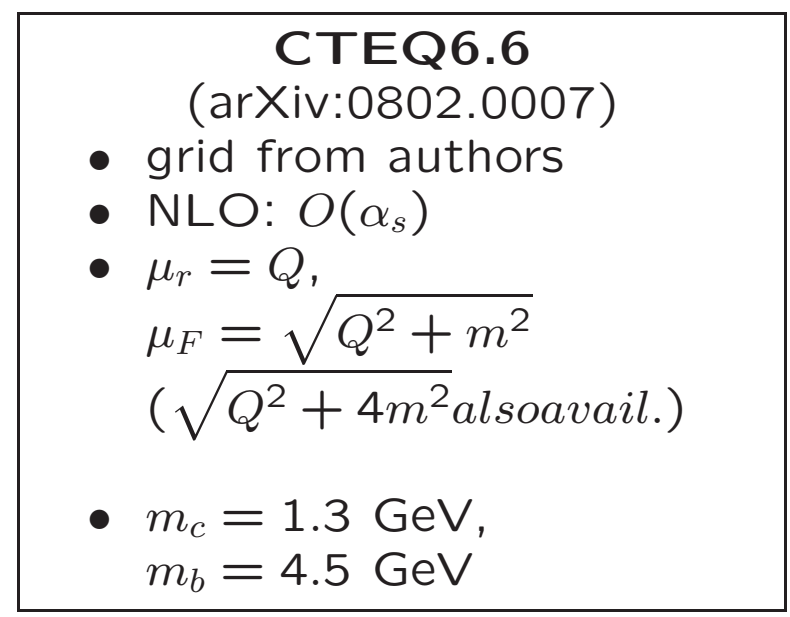

\section{ZM-VFNS}

\section{NNPDF}

(arXiv:0808.1231)

- grid from authors

- NLO: $O\left(\alpha_{s}\right)$

- $\mu_{R}=\mu_{F}=Q$

- $m_{c}=1.414 \mathrm{GeV}$, $m_{b}=4.3 \mathrm{GeV}$ 


\section{Charm NLO}
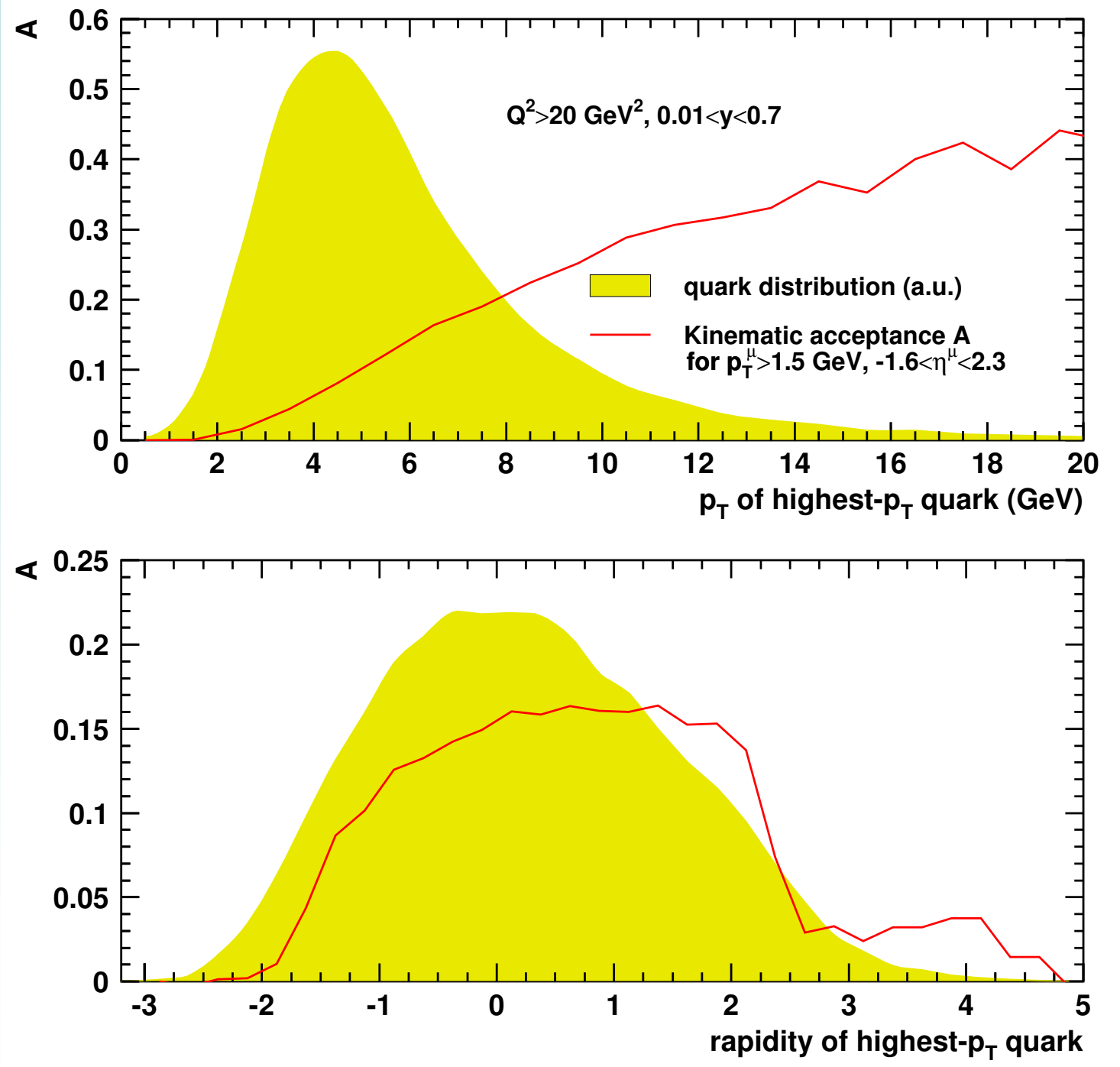

A becomes sizeable when $A>0.25<A>$

For charm:

$<A>\sim 13 \%$

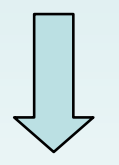

One of the quarks with $\mathrm{p}_{\mathrm{T}}>3 \mathrm{GeV}$ and $-1.5<\eta<2.5$ 


\section{ZEUS}
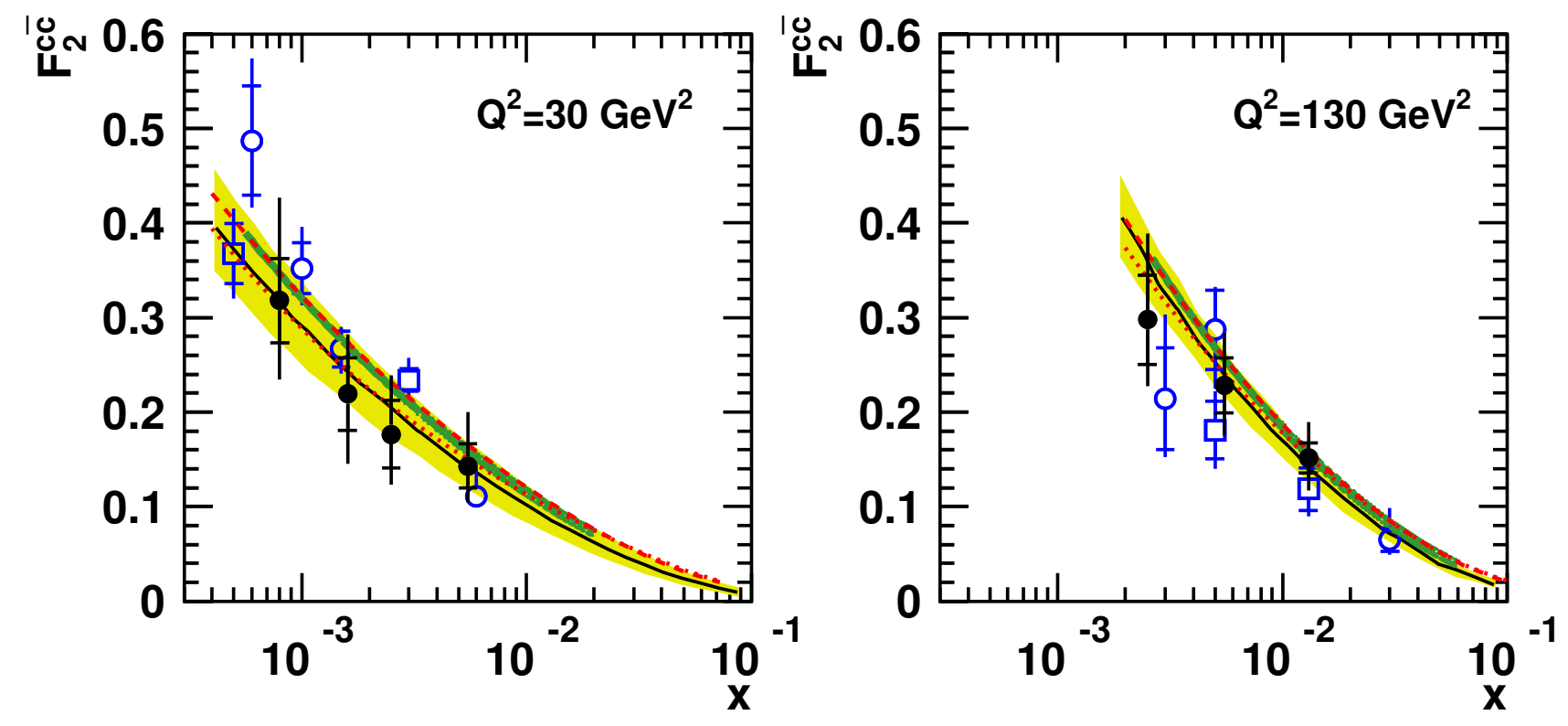

\section{$F_{2}^{c c}(\mathrm{x}):$}

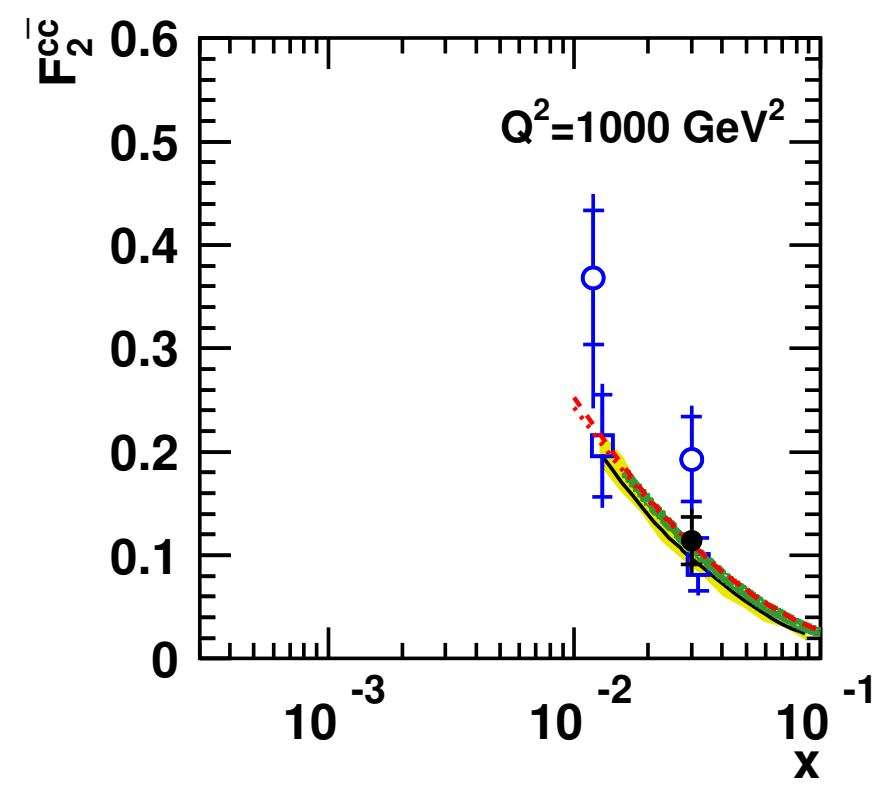

\section{published data}

vS

Charm

- ZEUS 126 pb $^{-1}(\mu)$

○ ZEUS $82 \mathrm{pb}^{-1}\left(\mathrm{D}^{\star}\right)$

ㄷ H1 $57 \mathrm{pb}^{-1}$ (VTX)

$\because$ ZEUS-S FFNS

- CTEQ6.6

. - MSTW08 nlo

..... MSTW08 nnlo different theories 


\section{ZEUS}

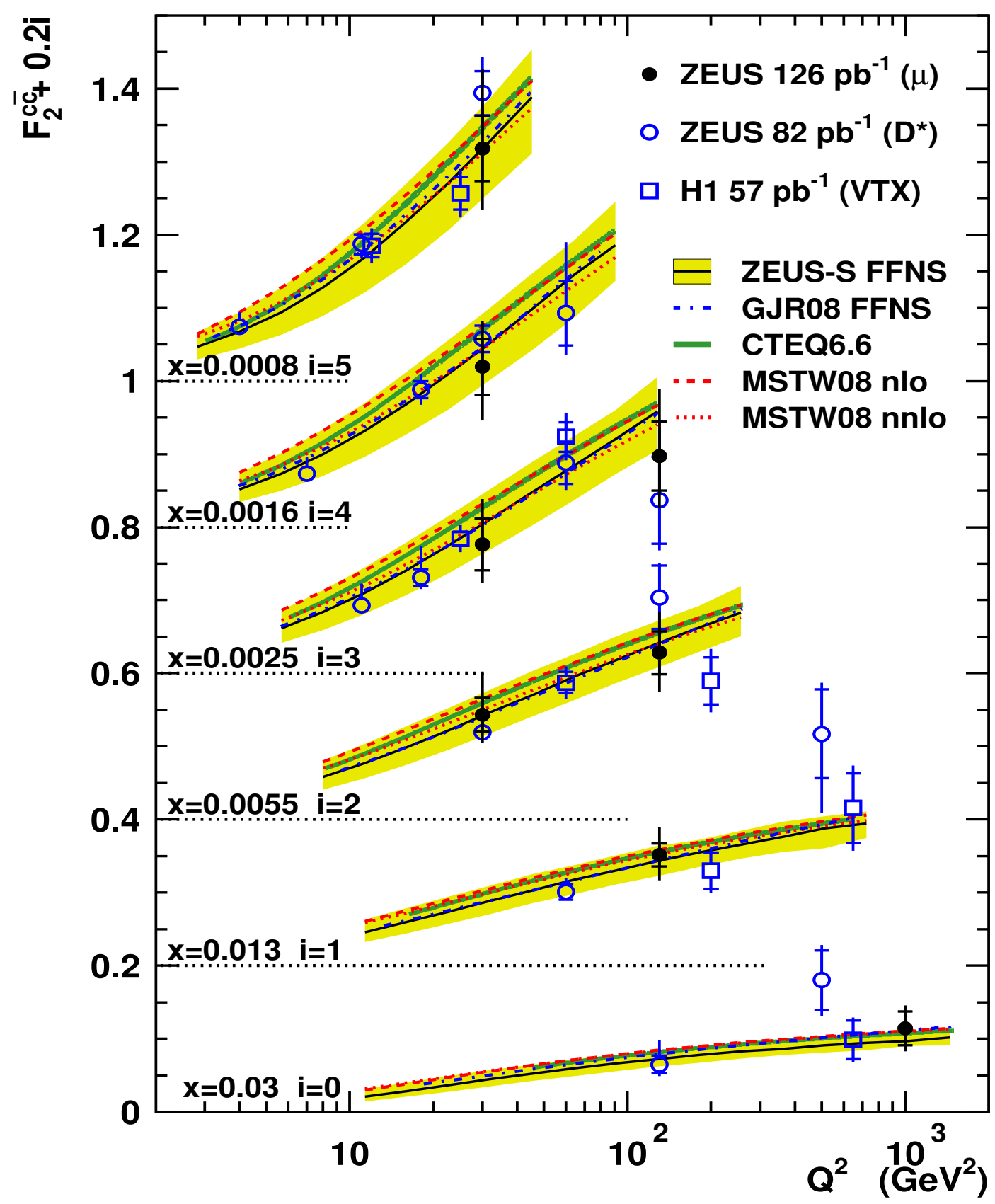

\section{$F_{2} c c$ (publ.+prel.)}




\section{Adding NNPDF}
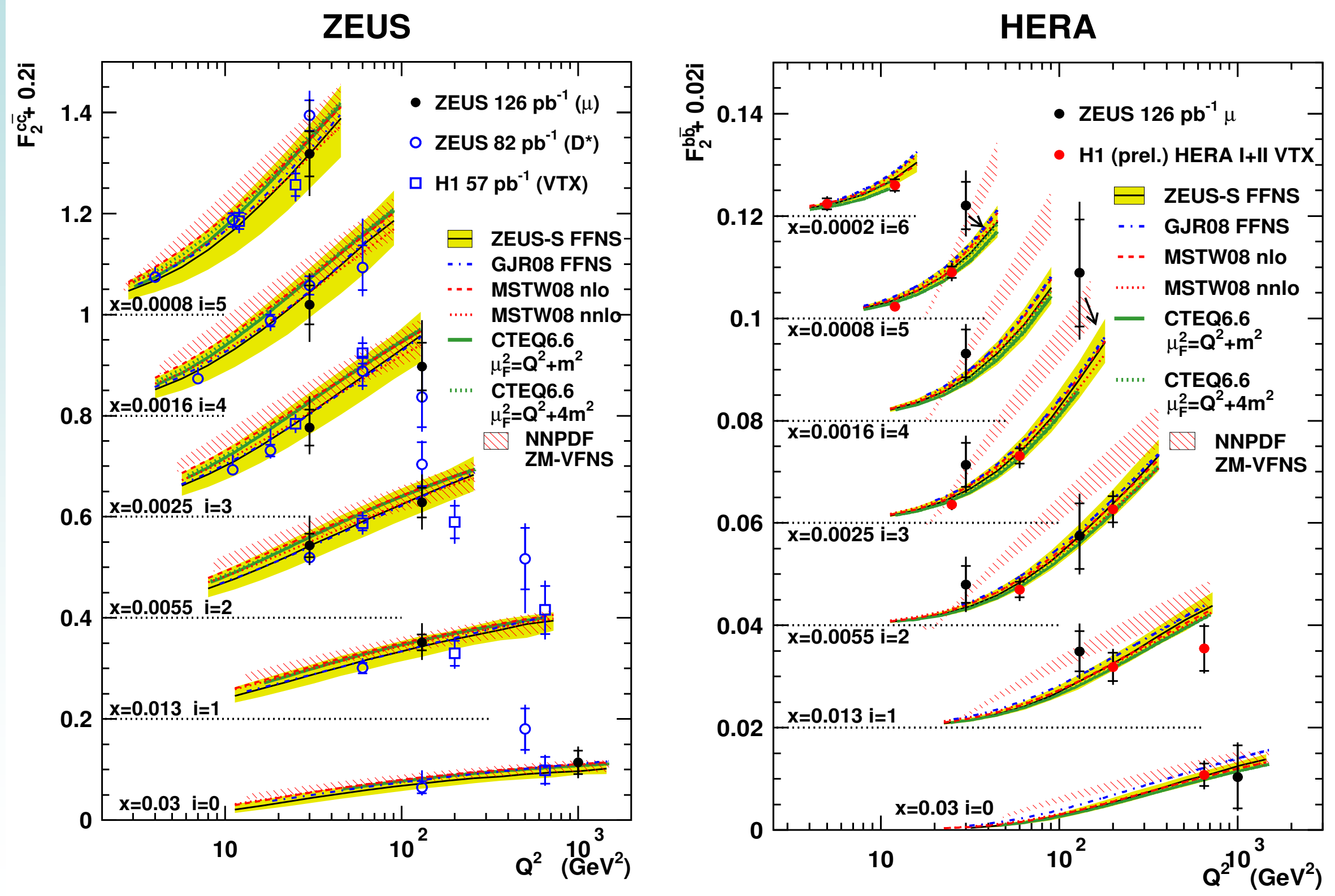